\title{
Pain and Disability in Osteoarthritis: A Review of Biobehavioral Mechanisms
}

\author{
Joost Dekker, ${ }^{1,4}$ Bob Boot, ${ }^{2}$ Luc H. V. van der Woude, ${ }^{2}$ \\ and J.W. J. Bijlsma ${ }^{3}$
}

Accepted for publication: April 16, 1991

\begin{abstract}
Pain and disability are cardinal symptoms in osteoarthritis. The literature is reviewed in order to identify causes of these symptoms at the articular, kinesiological, and psychological level. It is concluded that pain and disability are associated with degeneration of cartilage and bone (articular level), with muscle weakness and limitations in joint motion (kinesiological level), and with anxiety, coping style, attentional focus on symptoms, and possibly depression (psychological level). Biobehavioral mechanisms of pain and disability which explain the observed associations are described and the empirical evidence for these mechanisms is evaluated. Methodological and conceptual deficiencies in the research reviewed are pointed out and suggestions for further research are given.
\end{abstract}

KEY WORDS: osteoarthritis; pain; disability; review.

\section{INTRODUCTION}

Pain and functional disability are cardinal symptoms of patients with osteoarthritis (OA). As no causal therapy is available, treatment is primarily aimed at alleviation of these symptoms and prevention of their deterioration. Apart from drugs and physical modalities (e.g., local heat), therapeutic approaches include exercise and psychological interventions (Lorig et al.,

\footnotetext{
${ }^{1}$ Netherlands Institute of Primary Health Care, P.O. Box 1568, 3500 BN Utrecht, The Netherlands.

${ }^{2}$ Faculty of Human Movement Sciences, Free University, Amsterdam, The Netherlands.

${ }^{3}$ Department of Rheumatology, University of Utrecht, Utrecht, The Netherlands.

${ }^{4}$ To whom correspondence should be addressed.
} 
1987). A sound application of the latter approaches requires knowledge of kinesiological (e.g., impaired muscle function) and psychological (e.g., anxiety) determinants of pain and disability in OA. It should be known whether and by means of which mechanisms kinesiological and psychological factors cause pain and disability in OA patients. Knowledge of how these kinesiological and psychological determinants relate to articular determinants of pain and disability in OA is also required (i.e., pathological changes in cartilage and bone).

There are a number of studies which address these issues, but the evidence is scattered throughout the literature. The present review aims at an integration of the existing knowledge of articular, kinesiological, and psychological causes of pain and disability in OA. The empirical evidence is reviewed in the context of biobehavioral theories on pain and disability in related conditions (e.g., back pain and rheumatoid arthritis). Preceding the review, OA and its symptoms are briefly described.

\section{Osteoarthritis}

Osteoarthritis (or osteoarthrosis or degenerative joint disease) is a group of diseases characterized by two major morphological changes: (a) disintegration of articular cartilage and (b) formation of new bone. A series of events partially understood results in a loss of cartilage and degeneration of its biomechanical qualities. Cysts and sclerosis (thickening of bone) develop underneath the cartilage and new bone is formed at the margins of the bones ("osteophytes"). Neither the sequence nor the interrelationship of these changes in cartilage and bone is presently known. As the disease progresses, other tissues - synovial membrane, fibrous capsule, and associated musculature - also become affected (Brandt, 1985a, b; Threlkeld and Currier, 1988).

A distinction has been made between primary and secondary OA. In primary (or idiopathic) OA there are no known predisposing factors, while in secondary OA a certain factor (e.g., a structural abnormality) predisposes the individual to OA. The value of this distinction can be questioned, because in primary OA the predisposing factor may have been overlooked (Brandt, 1985b; Moskowitz, 1985). OA is an end-stage condition of multifactorial origin.

There is a strong association between age and the presence of OA. Radiological studies have shown a very low prevalence of osteoarthritic changes at age 30: less than $5 \%$ of the population show osteoarthritic changes (OA 
grades 3 and 4; the grading system is explained in the next section); at age 65 , at least one joint group is affected (grades 3 and 4 ) in at least $50 \%$ of the population. At more advanced ages the prevalence of OA increases further (Kirwan and Silman, 1987). Fortunately not all subjects experience symptoms: It has been estimated that about $30 \%$ of subjects with marked radiological OA will experience symptoms (Cobb et al., 1957). The most commonly involved joints are those of the fingers (distal and proximal interphalangeal joints), hand (first carpometacarpal joint), foot (first metatarsophalangeal joint), hip, knee, and cervical and lumbar spine. One or more joints may be affected. The disease may run a progressive course, but this is not necessarily the case (Brandt, 1985b).

Symptoms, Signs, and Consequences. Pain is the principal symptom of OA. At first it occurs after use of the joint and is relieved by rest. In later stages of OA pain may be present during rest and even sleep. Other symptoms of OA include stiffness following rest and instability of the joint (Brandt, 1985b; Moskowitz, 1985). On physical examination tenderness, crepitation, enlargement, deformity, and inflammation of the joint may be found. Restriction of range of joint motion, muscle atrophy, muscle spasms, and flexion contractures are common findings in OA. At the level of activities and tasks the patient may show various disabilities, depending on the joint involved. In one study these disabilities could be grouped into five categories: mobility, bending down, dexterity, bending arm, and reaching up (Bradley et al., 1984). Finally, OA is associated with limitations in the performance of social roles. Reduction in the ability to work, the performance of household chores, shopping, leisure, and recreational activities have been documented (Pincus et al., 1987; Yelin et al., 1987).

\section{REVIEW}

\section{Outline}

Pain and functional disability were selected as the target symptoms in this review. Pain and disability are cardinal symptoms in OA and they represent the subjective and behavioral consequences of the disease, respectively. The causes of pain and disability are reviewed at three levels: articular, kinesiological, and psychological level. Relevant studies were selected by means of computerized searches in Medlars, Psychological Abstracts, and Cambridge Scientific Abstracts. In addition, manual searches were performed. 


\section{Articular Level}

Studies on the relationship among articular changes, pain, and disability are summarized in Table I. In most studies the articular changes are assessed by means of radiological examination. Narrowing of the joint space, presence of osteophytes, sclerosis, and cysts are the principal radiological findings. These findings can be used to grade the severity of OA (Kellgren and Lawrence, 1957, 1963) The grading ranges from 0 to 4 , with 0 indicating no osteoarthritic changes and 4 indicating severe changes. In most studies a single question or a clinical evaluation has been used to assess pain (Kellgren and Lawrence, 1952; Cobb et al., 1957; Lawrence et al., 1966; Acheson et al., 1970; Miller et al., 1973; Davis 1981; Feison et al., 1987; Massardo et al., 1989). In several recent studies pain has been assessed with standardized questionnaires and scales (Forman et al., 1983; Lichtenberg et al., 1984; Keefe et al., 1987a, b). Disability has been assessed with questionnaires and observation methods (Acheson and Ginsburg, 1973; Baron et al., 1987; Keefe et al., 1987a, b; Summers et al., 1988; Patrick et al., 1989).

Pain. In nine studies an association between the severity of radio-logical OA and pain has been found (Kellgren and Lawrence, 1952; Cobb et al., 1957; Lawrence et al., 1966; Miller et al., 1973; Gresham and Rathey, 1975; Davis 1981; Forman et al., 1983; Felson et al., 1987; Patrick et al., 1989). This association has been found in all joints studied, with the exception of the lumbar and possibly cervical spine. Although radiological $O A$ and pain are associated in most joints, the association is not very strong. For example, Davis (1981) reported that the probability of reporting knee pain increased by about 0.21 when radiological evidence of $O A$ in the knee was present (in an analysis controlling for sex, current physician treatment, and the interaction of these variables; the interaction of radiological OA and treatment added another .05 to the probability of reporting pain).

In five other studies no relationship between radiological OA and pain was found (Acheson et al. 1970; Lichtenberg et al., 1984; Keefe et al., 1987a, b; Summers et al., 1988). In addition, one study reported the absence of an association between changes in the severeness of radiological OA and changes in pain over time (Massardo et al., 1989). Again, this result shows that the association of radiological OA and pain is very weak. Possible explanations are discussed in the next section, but there is a methodological problem as well. The nine studies which demonstrated an association between pain and radiological OA were all - with one exception - population based or they used an appropriate control group. All except one of the six studies which failed to find an association used patients as subjects. Most probably there is a restricted range of pain in patients: Subjects with no or minimal pain are very rare in clinical studies, because subjects without pain are not expected to 
seek treatment. With a restricted range it is difficult to demonstrate a relationship between variables. This is probably one of the factors which may account for the lack of association in the clinical studies.

Mechanisms of Pain. Lawrence et al. (1966) noted that radiological OA is not a sufficient cause of pain. Cartilage does not have a nerve supply. This means that changes in articular cartilage do not directly contribute to nociception. Therefore, radiological signs of cartilage changes cannot be expected to be strongly associated with pain. This explains some of the discrepancy between radiological $O A$ and pain. It should also be noted that radiological assessment provides only an indirect measure of the state of cartilage: On a radiograph, narrowing of the joint space due to marked degeneration of cartilage can be observed, but this is not a direct measure of the extent of fibrillation of cartilage. However, because of the lack of innervation, even a direct measure of the state of cartilage is not expected to show a strong association with pain.

Kellgren (1983) has suggested that the subchondral bone is an important source of pain in OA. Subchondral bone is innervated and the active remodeling of bone (osteofytes) may result in a increased sensitivity of the nociceptors. Pressure on exposed subchondral bone, sclerosis, and cysts may contribute to pain. Osteophytes may also irritate adjacent soft tissues such as ligaments, tendons, and periost (Threlkeld and Currier, 1988). In addition to the subchondral bone Kellgren suggested two other important peripheral sources of pain. One is ligamentous sprain, which is dealt with in the next section. The other is inflammation, primarily of the synovium. But neither irritation of soft tissues nor ligamentous sprain nor inflammation is detected on radiological examination. Thus, the weak association of radiological OA and pain seems to be explained largely by a discrepancy between the articular changes detected on radiological examination and the process of nociception: (a) radiological examination does (indirectly) concern changes in cartilage, but cartilage does not directly contribute to nociception; (b) among the articular changes which do contribute to nociception, only the changes in subchondral bone are assessed on radiological examination.

Disability. The relationship between disability and radiological OA has been studied in patients with OA of the knee, feet, and hand (including wrist) (see Table I). In one study an appropriate control group was used (Patrick et al., 1989) and one study was population-based (Baron et al., 1987). In both studies a significant association was found between disability and radiological OA. Five other studies only used patients or symptomatic subjects: In four studies some association between radiological $\mathrm{OA}$ and disability was found (Acheson and Ginsburg, 1973; Miller et al., 1973; Keefe et al., 1987a, b; Summers et al., 1988). Thus, even in clinical studies the relationship of radiological OA to disability can be demonstrated. But, 


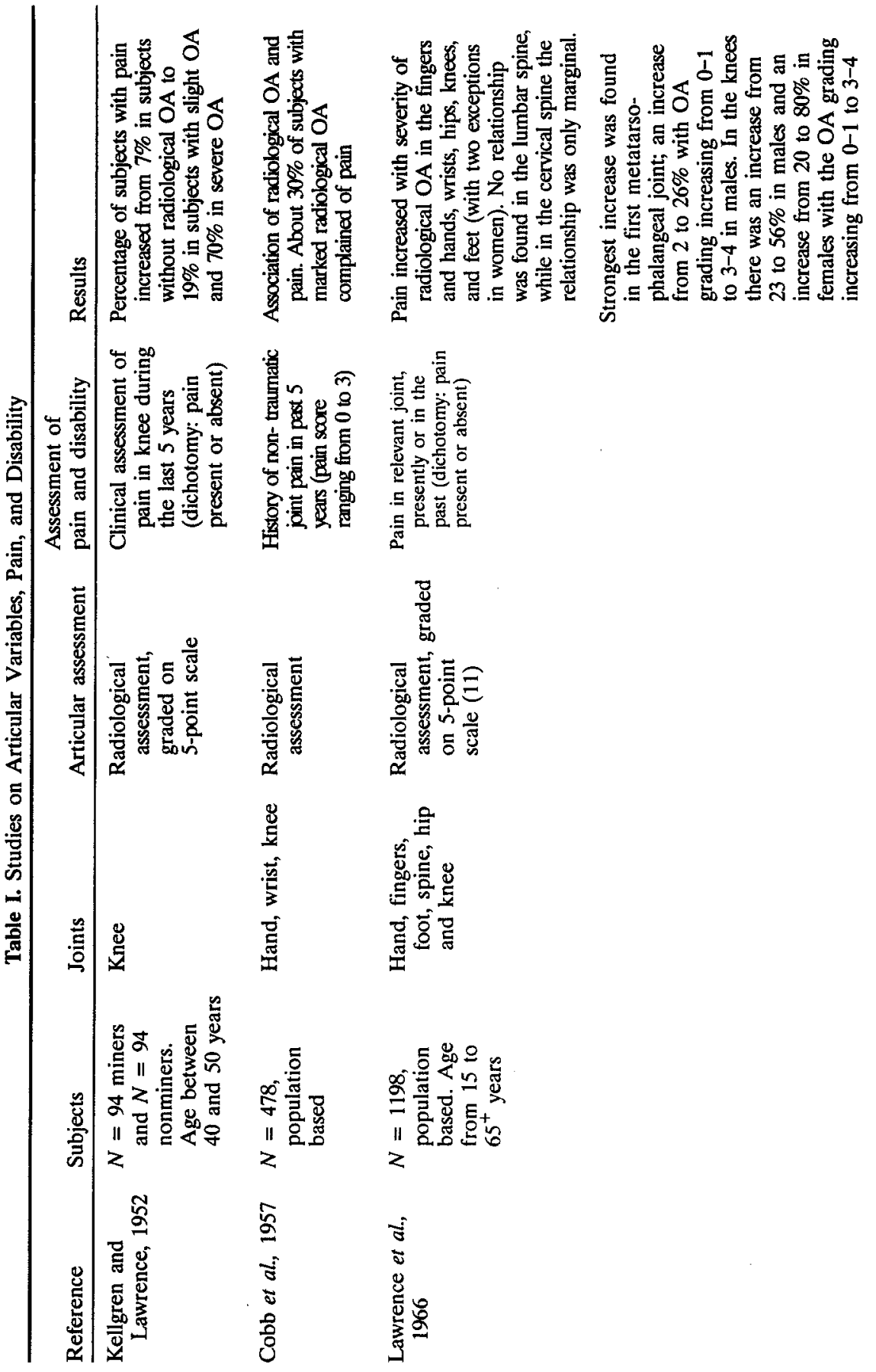



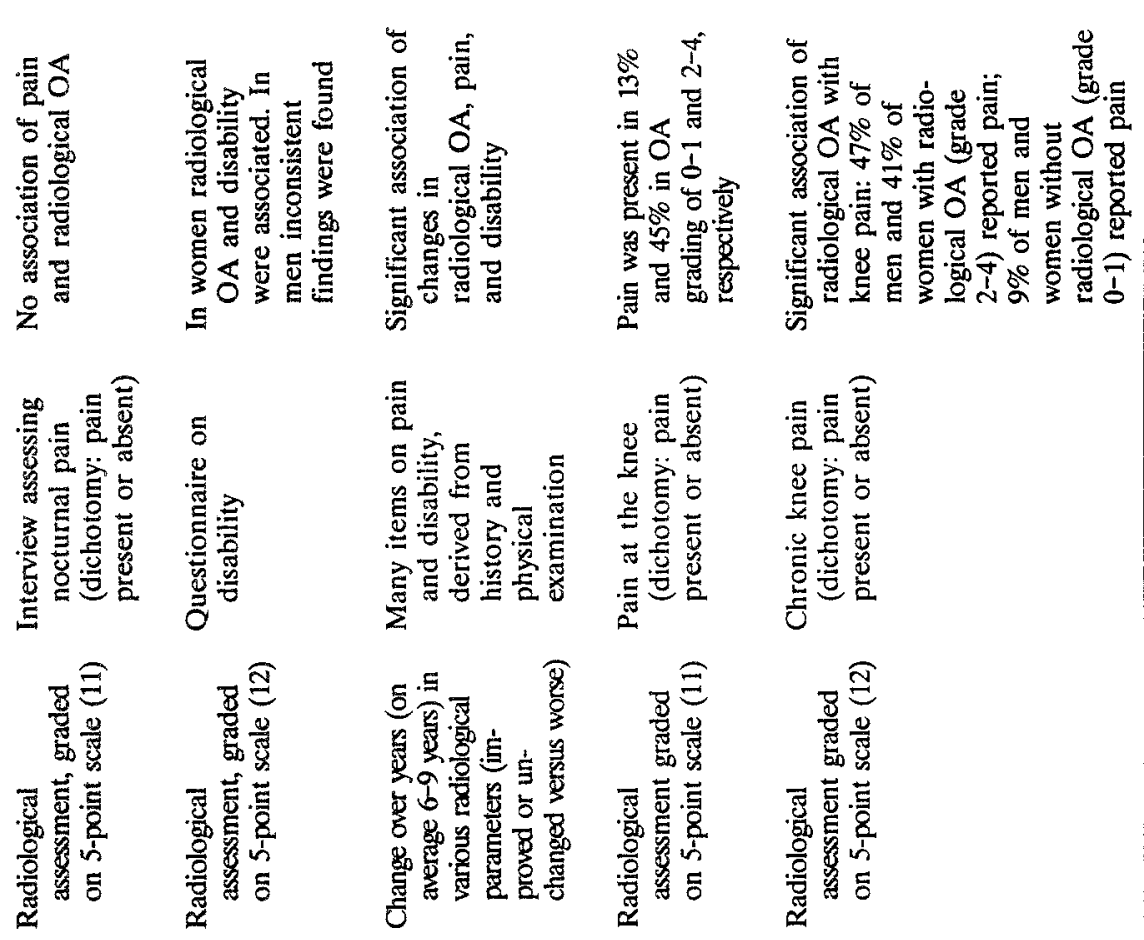

蓠

总僖

巡

$\stackrel{\Xi}{5}$

$\stackrel{8}{5}$

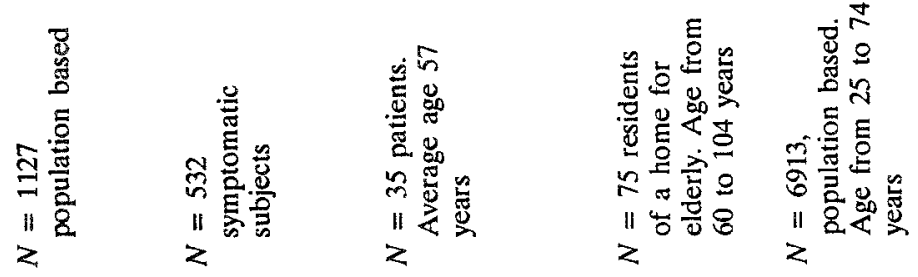

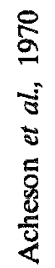

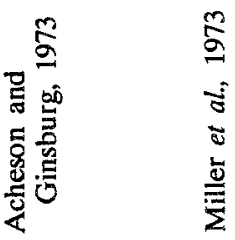

总

$\frac{\bar{\alpha}}{\stackrel{0}{2}}$ 


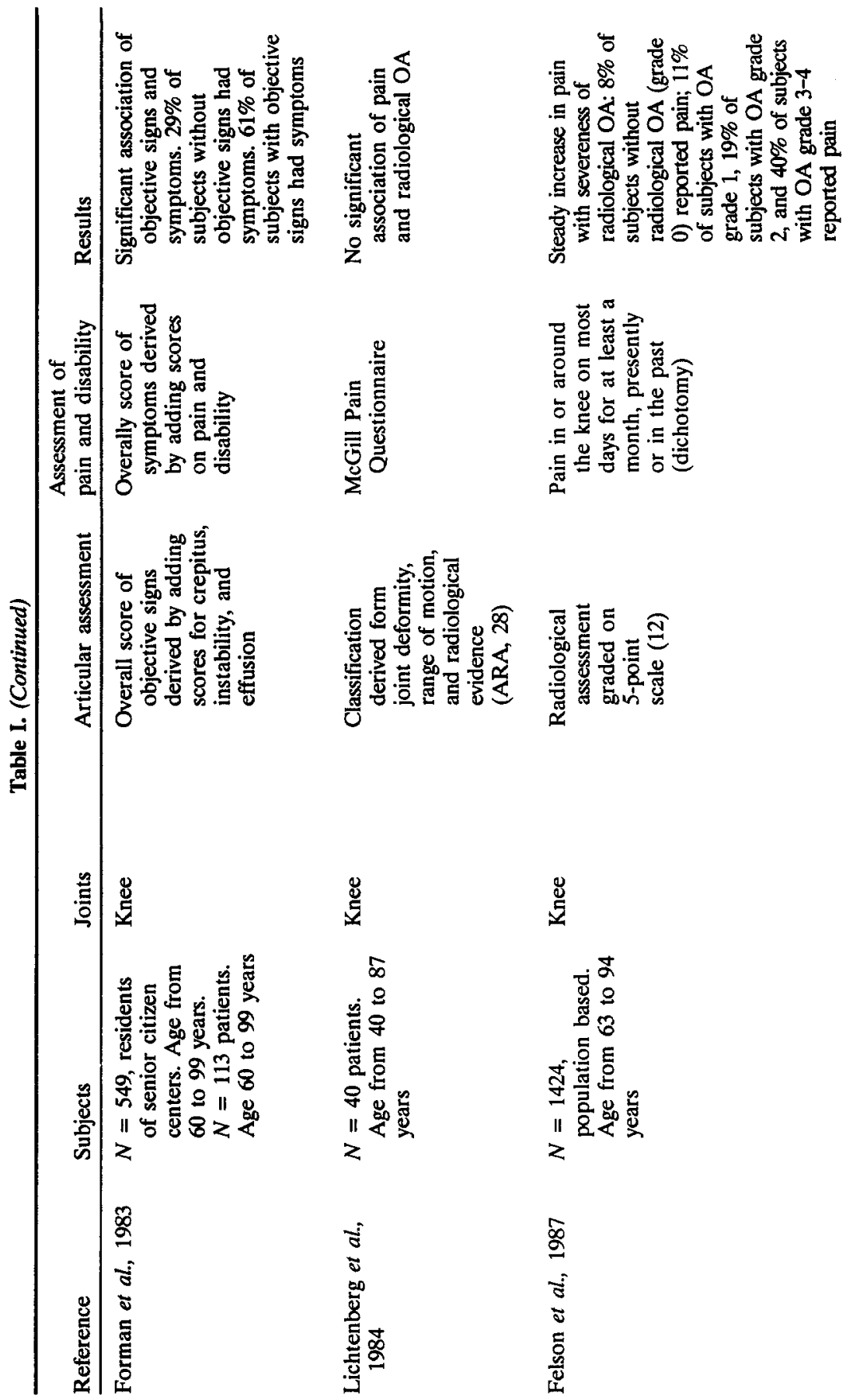



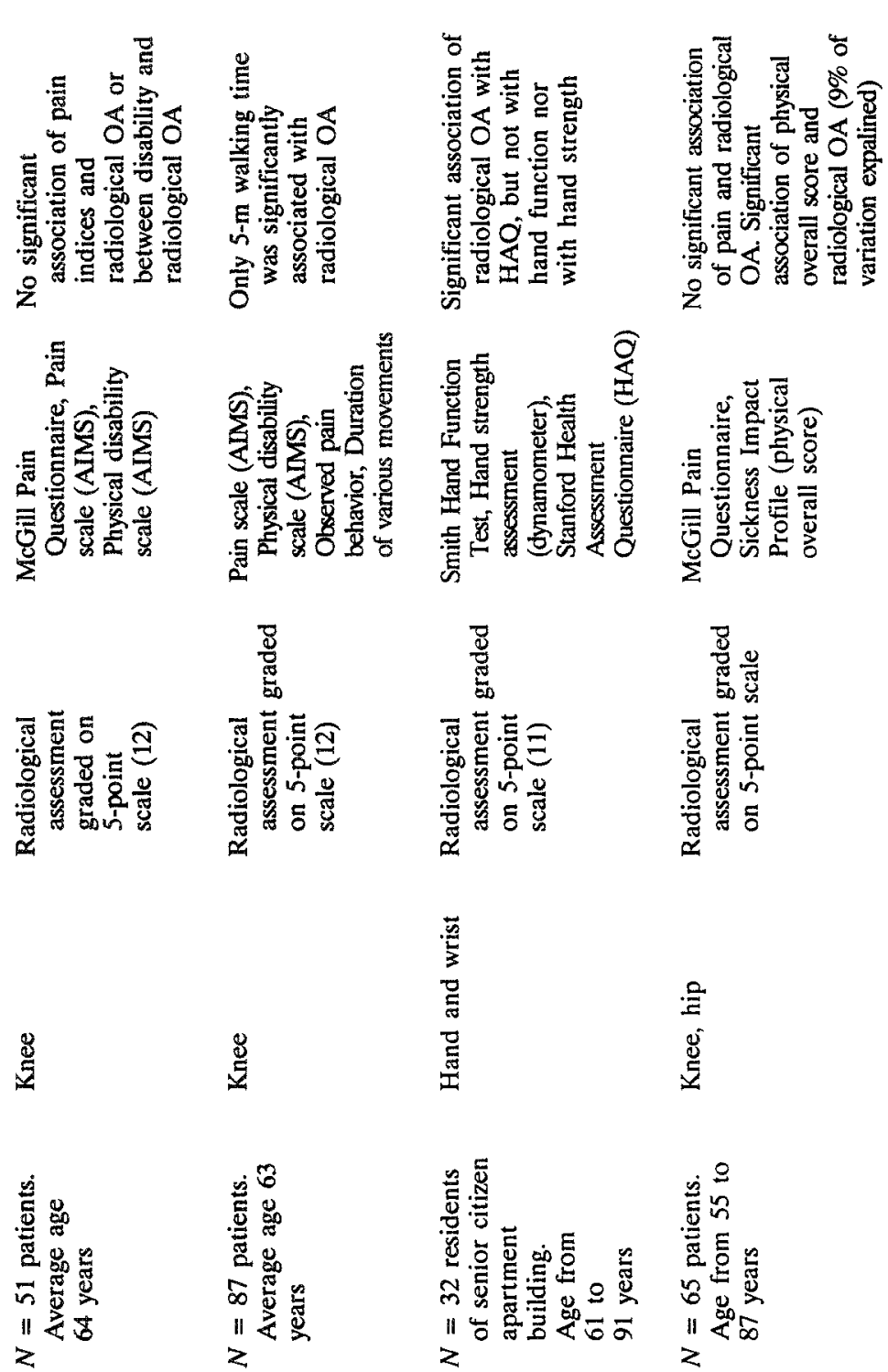

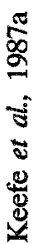

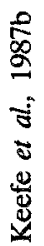

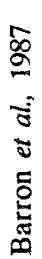

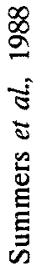




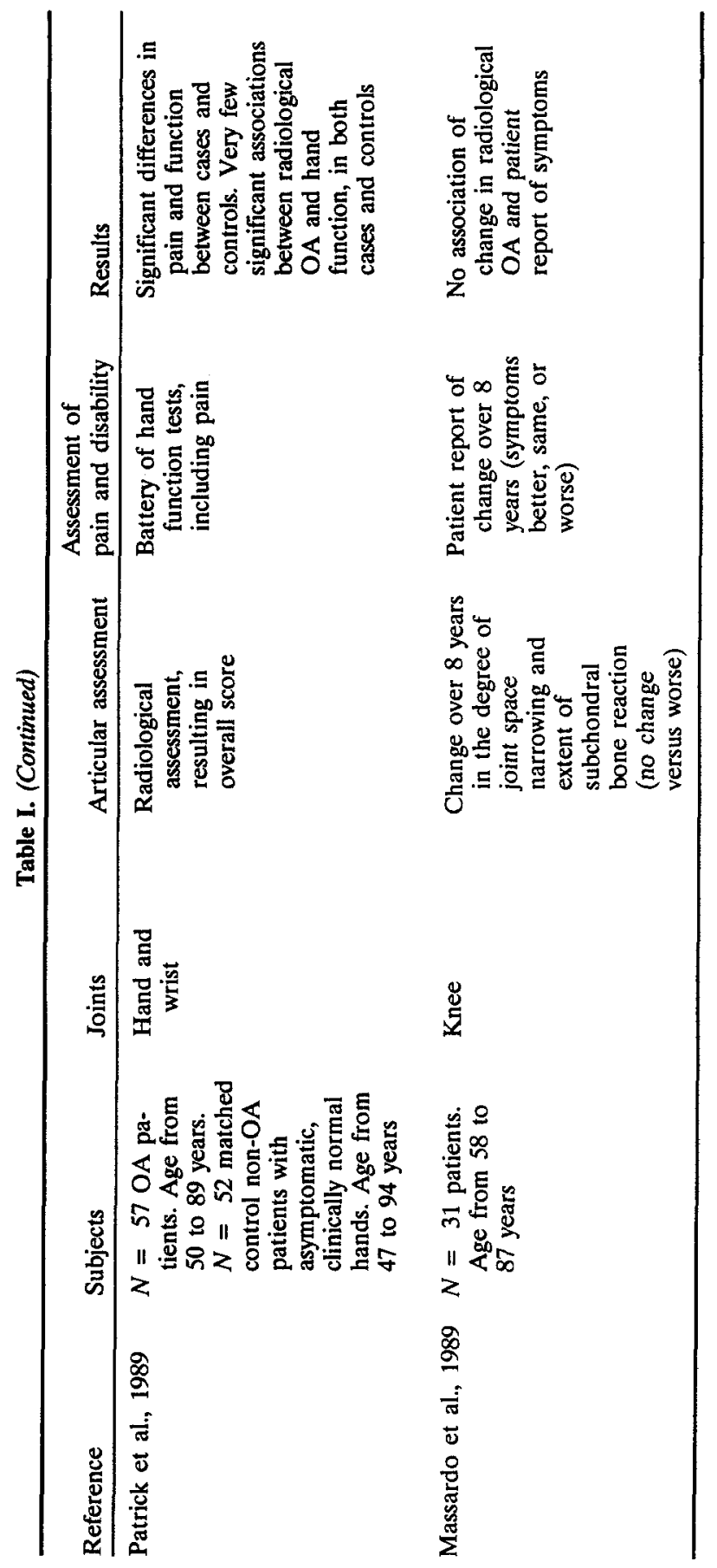


again, this relationship is not very strong. For example, in the controlled study on OA of the hand (Patrick et al., 1989), patients had more difficulty in completing tasks, used more trick movements, and had reduced grip strength, but they were similar to controls in being able to complete the tested tasks. And in the clinical studies, only a few test items showed an association between radiological $\mathrm{OA}$ and disability.

Mechanisms of Disability. The association of disability and radiological $\mathrm{OA}$ seems to be caused by mechanical limitations of the joint. OA causes incongruity of joint surfaces and this results in limitation of joint motion and in disability. Similarly, osteophytes and intraarticular loose bodies may limit joint motion, which leads to disability (Brandt, 1985b).

\section{Kinesiological Level}

As OA progresses the articular capsule and associated musculature become affected (Brandt, 1985b; Threlkeld and Currier, 1988; Moskowitz, 1985). In the capsule both laxity and contractures can be found. The muscular manifestations include both muscle weakness and muscle spasm. Nordesjö et al. (1983) made a comparison between patients with severe $\mathrm{OA}$ of the knee and a reference group. On extension and flexion of the knee patients had only $55-70 \%$ of the muscle strength of the reference group. Lankhorst et al. (1985) compared osteoarthritic knees with normal knees of the same subjects: On extension and flexion muscle strength on the affected side was $65-83 \%$ that on the normal side. Beals et al. (1985) also reported that muscle strength on knee flexion was significantly less in OA knee patients than in matched controls.

The relationship of muscle function and joint motion to pain and disability has been studied. Relevant studies have been summarized in Table II. The studies on muscle function are discussed first.

Pain, Disability, and Muscle Function. Lankhorst et al. (1985) tested muscle strength in OA knee patients. The testing of muscle strength involved the measurement of the torque, which the patient is able to generate during extension and flexion of the knee. It was also determined whether the patient had experienced knee pain during the past week and whether the patient experienced pain during the testing of muscle strength. The best predictor of knee pain during the past week was pain experienced during muscle testing (23\% of variation explained), followed by knee extension strength (9\%). Disability - assessed with both a questionnaire and standardized tests - was best predicted by knee extension strength (21$33 \%$ of variation explained). Pain during muscle testing explained another $2-6 \%$ of variation in disability. Thus, this study demonstrated a substantial 


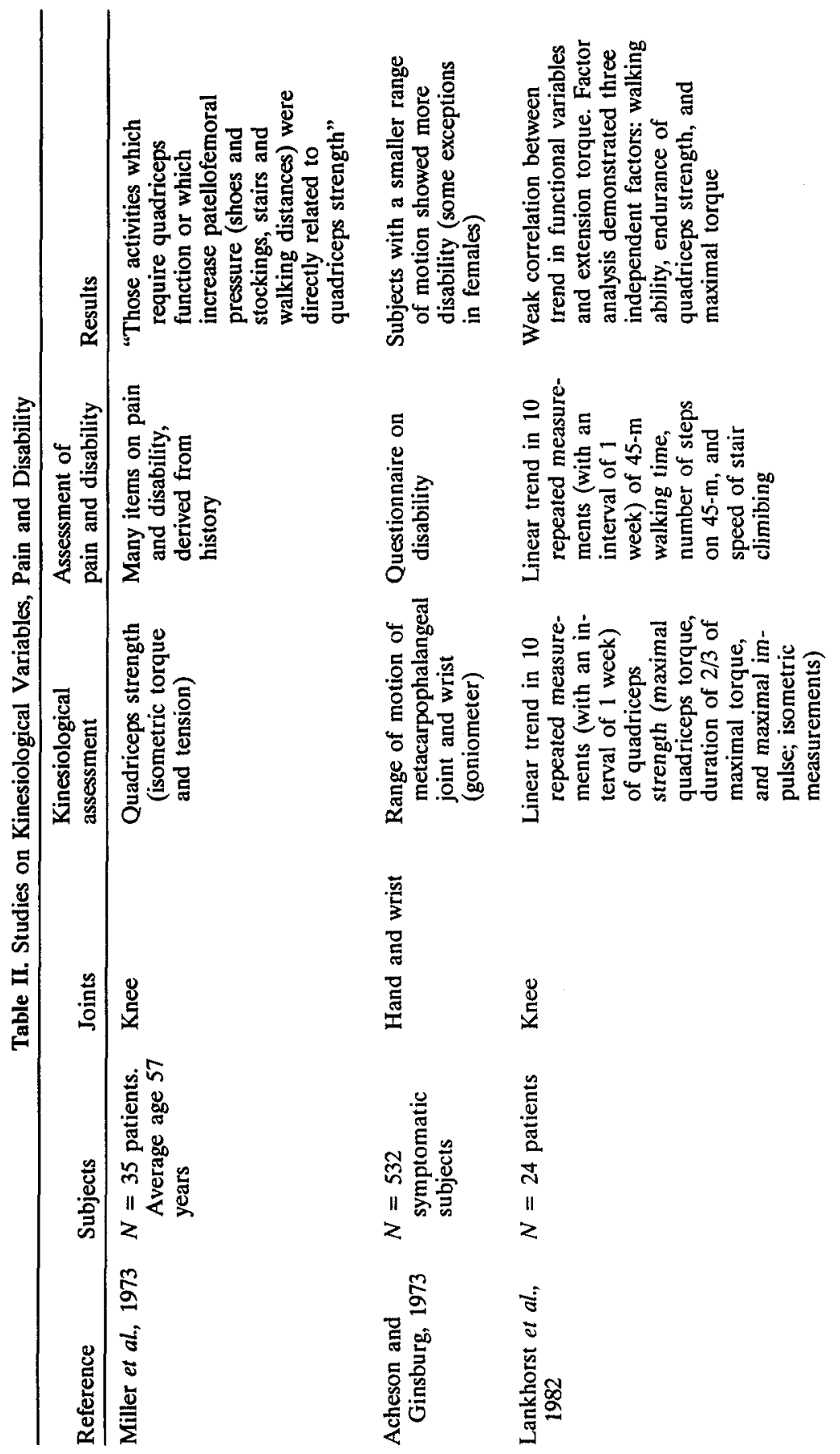



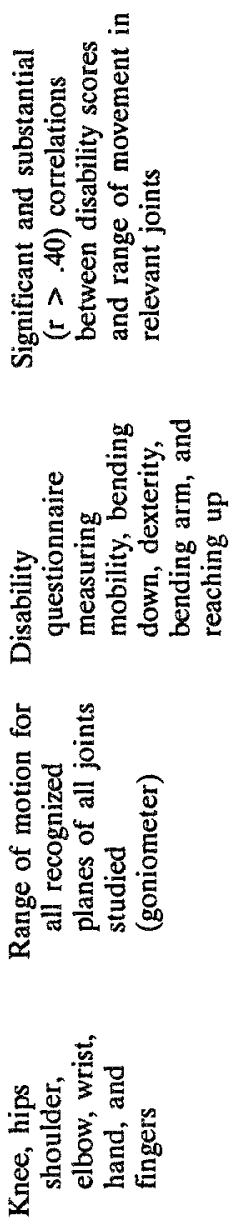

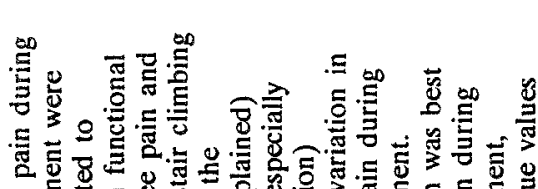



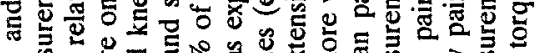

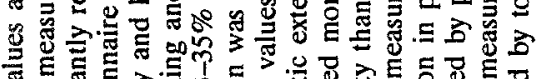
贸

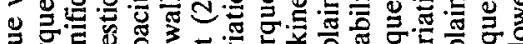

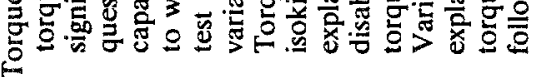



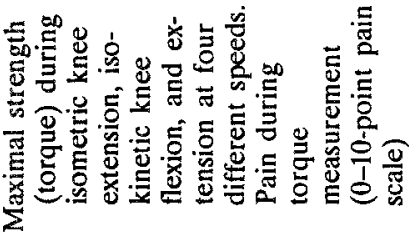

$\stackrel{\Xi}{\square}$

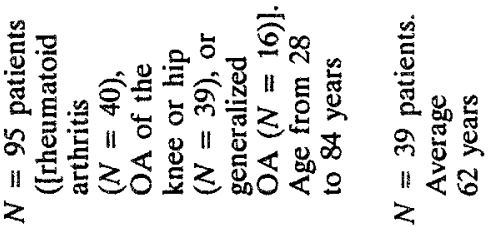

兽

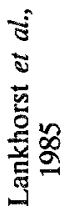




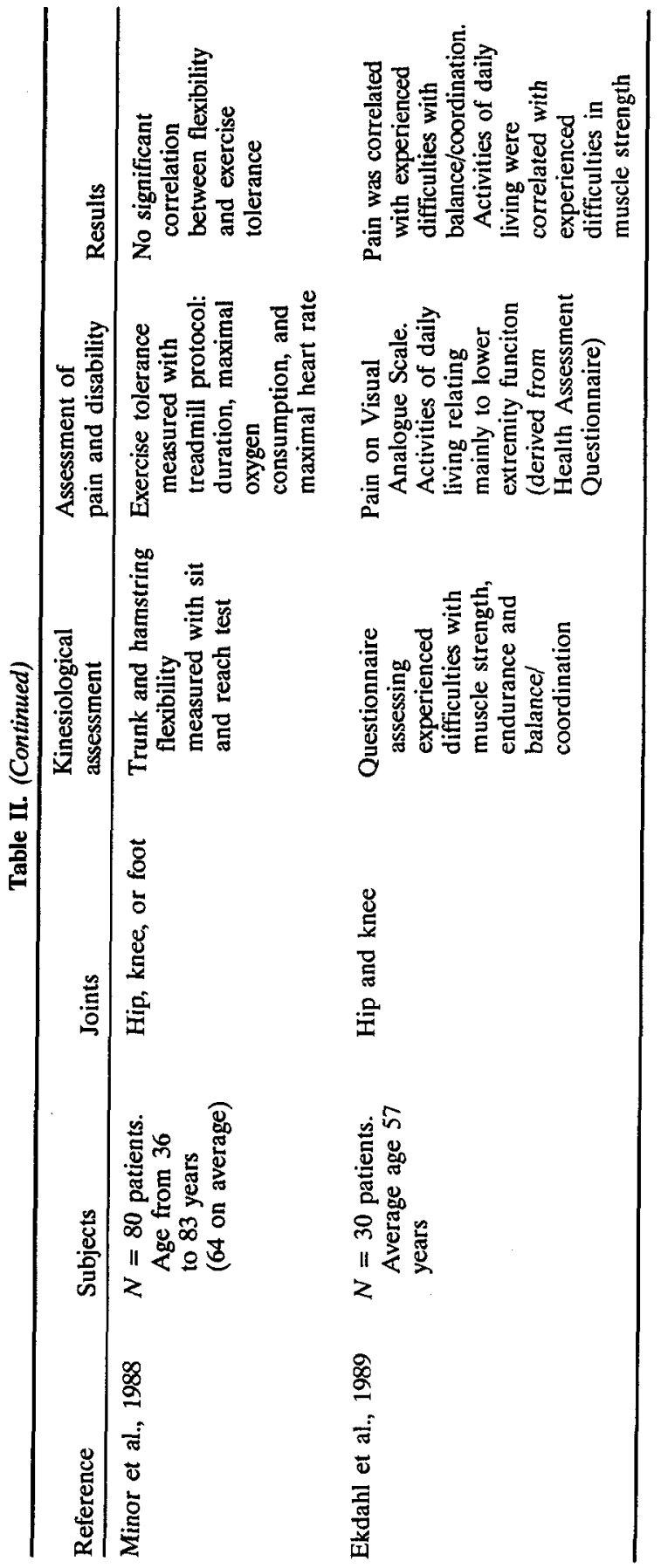


negative association of muscle strength and disability in $\mathrm{OA}$. There is also a negative association between muscle strength and pain, but this association is somewhat less pronounced.

Miller et al. (1973) also reported that muscle strength and disability are associated; however, this study lacks specific information, which makes it very difficult to evaluate the results. Lankhorst et al. (1982) determined the relationship between changes over time in muscle strength (torque) and changes in disability. Patients were tested 10 times at intervals of 1 week. It was concluded that no association existed between changes in muscle strength and disability. However, the analysis of change scores is a very indirect approach; unfortunately, direct correlations between muscle strength and disability were not reported by these authors. Ekdahl et al. (1989) using questionnaires - reported that experienced difficulties with muscle performance were associated with pain and with disability. Inspection of the items they used suggests that the questionnaires on disability and on muscle performance measure more or less the same things. Thus, in the present context, only the correlation between muscle performance and pain is relevant. In evaluating the results of these last three studies it appears that the studies by Miller et al. (1973) and Ekdahl et al. (1989) give some support to the conclusion derived from the Lankhorst et al. (1985) study: muscle strength is negatively associated with disability and - somewhat less strongly - with pain in OA patients.

Mechanisms of Pain and Disability Involving Muscle Performance. Various theoretical models on muscular determinants of pain and disability have been hypothesized (see Flor and Turk, 1984; Turk and Flor, 1984; Stokes and Young, 1984; Dolce and Raczynski, 1985; Keefe and Gil, 1986). The model based on the biomechanical effects of muscle weakness (cf. Dolce and Raczynski, 1985; Keefe and Gil, 1986) seems to offer the best explanation for the data on muscle performance which were summarized above. According to this model muscle weakness or asymmetric muscular activity produces unstable joints. Stress on unstable joints leads to strain in innervated tissues (capsule and ligaments, in particular) and thus to pain and disability. Avoidance of activity results in disuse of the muscles, which enhances muscle weakness. This starts a vicious circle of disuse, muscle weakness, pain, and disability. It should be noted, however, that the present data provide only a rather rough test of this model. Only a cross-sectional association has been demonstrated, while the hypothesized vicious circle of disuse, muscle weakness, pain, and disability has not been tested. It should also be noted that muscle weakness may be caused by factors other than disuse such as reflex inhibition (Threlkeld and Currier, 1988; Stokes and Young, 1984). 
Other theoretical models on muscular determinants of pain focus on muscle spasm, which leads to ischemia and pain (cf. Turk and Flor, 1984; Keefe and Gil, 1986). Although these models may be appropriate, there is no empirical evidence to support these models in OA. Thus, a biomechanical model seems to offer the best explanation for the observed association of muscle weakness, pain, and disability. But further research should be aimed both at more appropriate tests of this model and at tests of alternative models.

Disability and Joint Motion. Badly et al. (1984) determined correlations between range of motion (ROM) of various joints and disability in arthritis patients. As expected, high correlations (.40 and higher) occurred only between ROM of those joints which were relevant for certain aspects of disability. For example, range of knee and hip flexion were correlated with mobility and bending down, while range of shoulder abduction correlated with dexterity, bending the arm and reaching up. These correlations ranged from .42 to .78 . In addition to OA patients, patients with rheumatoid arthritis were also included in this study. Thus, there is a confusion of diagnoses in this study. Acheson and Ginsburg (1973) have reported an association between ROM of the knuckles and performance of various tasks in OA patients. Minor et al. (1988) reported that "trunk and hamstring flexibility" was not associated with exercise tolerance.

Mechanisms of Disability Involving Joint Motion. The results of the studies reported above indicate that limitations in ROM of a particular joint are associated with limitations in specific activities, while other activities and exercise tolerance are not affected. At the kinesiological level various mechanisms may cause reduced ROM and disability in OA. These mechanisms include capsular contractures, muscle contractures, and muscle spasms. On the basis of the studies reported above, it is not possible to determine which of these mechanisms is operating in OA.

\section{Psychological Level}

Studies on the relationship of psychological variables, pain, and disability are summarized in Table III. Psychological assessment concerned emotions, coping style, life events, and personality characteristics.

Pain and Disability. Lunghi et al. (1978) found an association of depression with pain and disability and an association of the level of unpleasant events with disability. These authors also analyzed the association over time of (un)pleasant events and symptoms. Surprisingly, it was found that a decrease in events (both pleasant and unpleasant) was followed by an increase in symptoms (i.e., pain and disability). In addition, an increase 
in symptoms was followed by a decrease in events. The authors suggested a distraction hypothesis, whereby a low level of events leads the individual to focus increasingly on his symptoms, which causes a heightened perception of symptoms. A heightened perception of symptoms subsequently leads the individual to reduce his or her activity and thus to a decrease of events.

Lichtenberg et al. (1984) reported an association between hypochondriasis and pain. However, they used the MMPI (Minnesota Multiphasic Personality Inventory) to assess hypochondriasis; it has been shown that elevated MMPI scores reflect somatic disease rather than psychological status (Pincus et al., 1986). This is due to certain MMPI items which evaluate symptoms (e.g., "I have little or no pain"). Findings based on the MMPI may therefore be artifacts. Summers et al. (1988) reported that depression, anxiety, and coping style were correlated with pain and disability. Depression was measured using the Beck Depression Inventory: This scale may produce the same kind of artifacts as the MMPI, which may be an explanation for the high percentage of explained variation reported in this study (Peck et al., 1989). Anxiety and coping style ("learned resourcefulness") also showed significant but much weaker correlations. The latter findings probably are not artifacts, because the scales which were used to measure anxiety and coping style do not evaluate symptoms.

The association of pain coping with symptoms has been studied by Keefe et al. $(1987 \mathrm{a}, \mathrm{b})$. These authors studied a coping style characterized by the perceived ability to control and decrease pain and by a low level of catastrophizing ("pain control and rational thinking"). This coping style was negatively associated with pain, self-reported disability, and observed disability. The association with observed disability is of much interest: The association between self-reported coping style and self-reported disability might be an artifact caused by subjective bias or response tendencies in the self-report (Watson and Pennebacker, 1989); but this criticism does not apply to observed disability. Accordingly, the association of coping style and disability seems to be real.

Mechanisms of Pain and Disability. The purpose of these studies was not to demonstrate a psychological effect on the onset of disease. These studies were designed to examine a possible relationship between psychological processes and variations in symptoms (pain and disability). It can be concluded that such a relationship has been demonstrated. However, there are some ambiguities in the interpretation of this relationship, because most studies only determined whether a correlation between psychological variables and symptoms exists. The absence of experimental or prospective designs precludes causal interpretations. The only exception is the study by Lunghi et al. (1978), which used a prospective design. These authors 


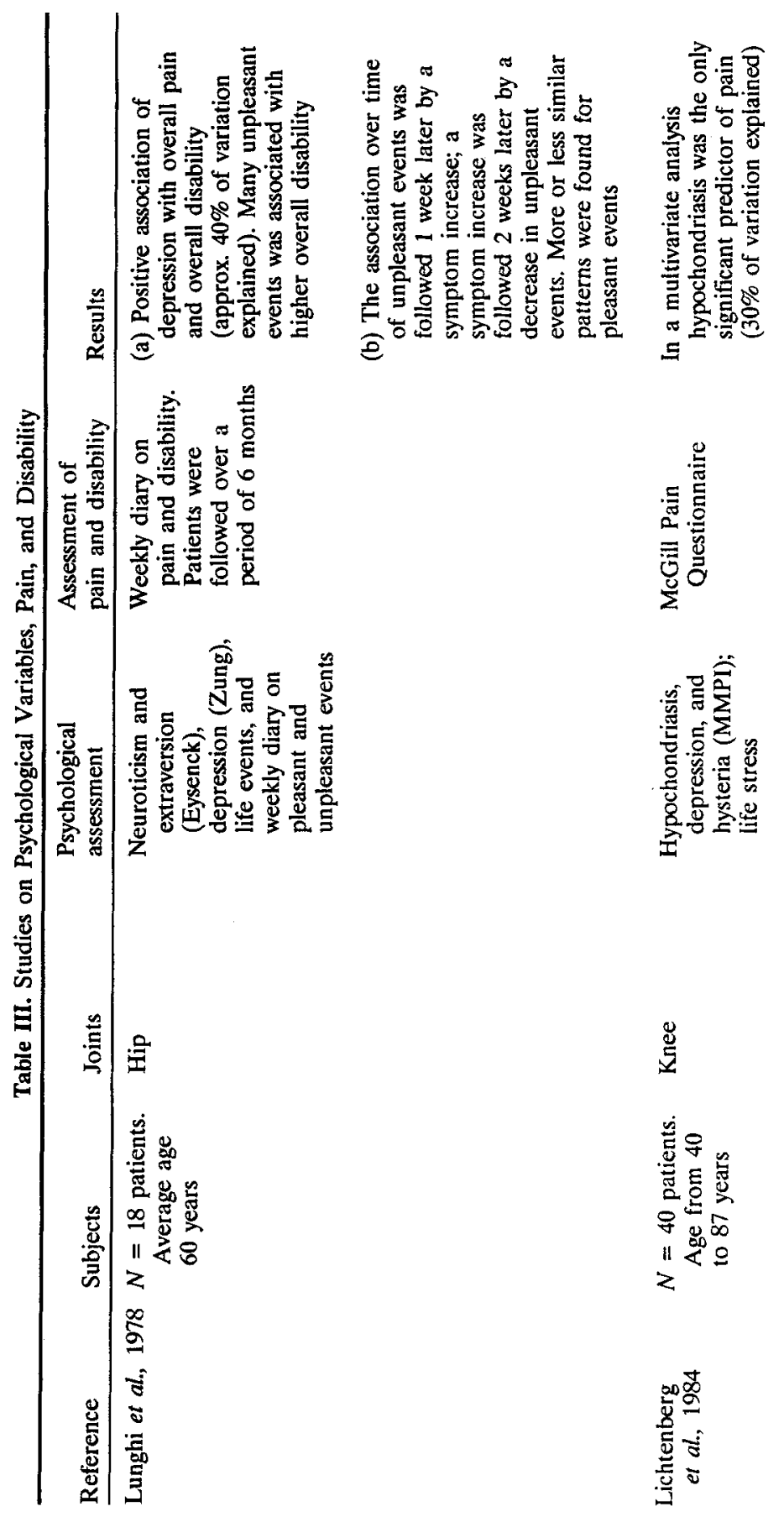




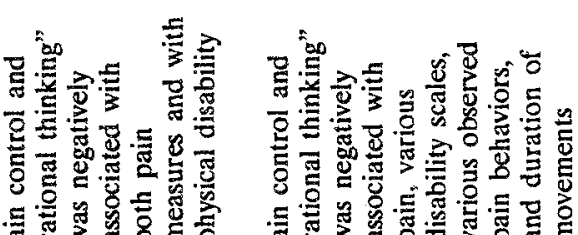

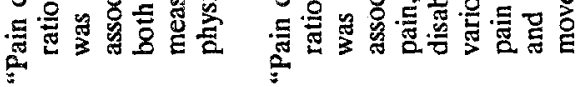

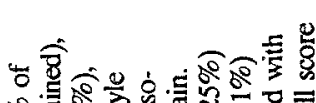

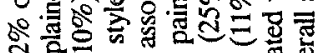
d

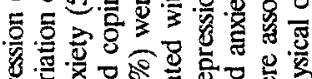

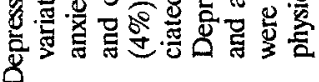
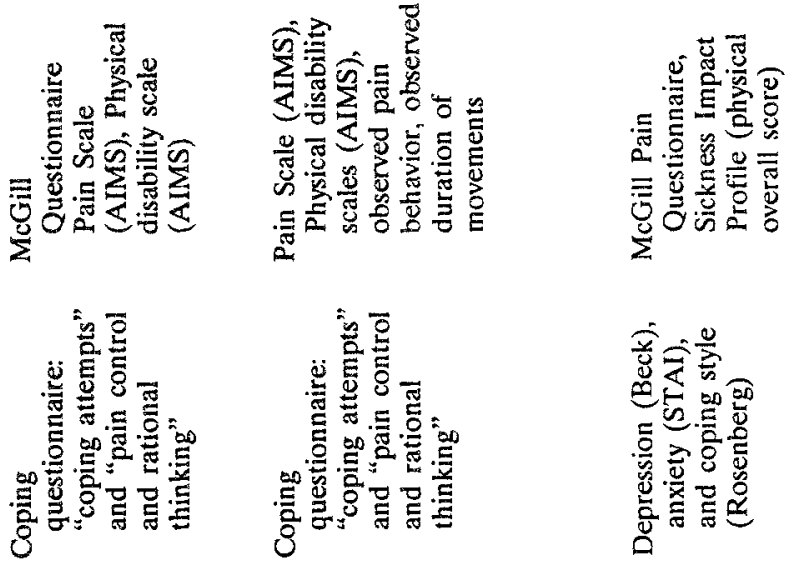

$\stackrel{8}{3}$

$\underset{\mathscr{Z}}{\mathscr{Z}}$

先
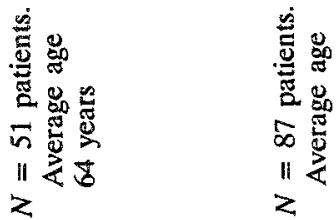

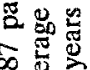

$\infty \frac{2}{0}$

$z$

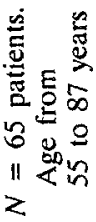

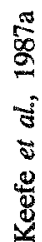

0
0
0
0
0
0
0
0
0
0

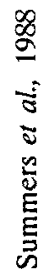


based the explanation of their unexpected findings on the concept of distraction: In the absence of distracting events the individual focuses on body sensations and this leads to an increase in experienced pain. And distraction from body sensations is hypothesized to reduce the experience of symptoms. In diseases other than OA, similar mechanisms have been hypothesized (Pennebaker, 1982; McCaul and Mallot, 1984; Barsky and Klerman, 1983).

In the other studies, the interpretation of the findings is less clear. Anxiety and coping style may lead to pain, but the reverse is also a valid explanation of the observed association. Possibly both effects occur. This type of bidirectional model of emotions and symptoms has been formulated by Pennebaker (1982), who hypothesizes that emotions and symptoms are integrated into a cognitive schema. Because of this integration emotional experiences lead to the experience of symptoms and vice versa.

However, this model does not explain the negative association of coping style and disability, in particular observed disability. This model concerns the experience of symptoms (such as pain): The cognitive schema leads to an association of the experience of symptoms and emotions. Pennebaker's model does not explain the association of emotional states (or coping) and overt behavior, such as observed disabilities. The concept of avoidance may offer a more appropriate explanation. According to this explanation, the patient avoids high levels of activity, because activity induces pain. An emotional or catastrophizing reaction to pain is expected to strengthen this avoidance of activity. The ensuing disuse of muscles and muscle weakness lead to disability. In other diseases similar mechanisms based on the concept of avoidance have been hypothesized (see Turk and Flor, 1984; Philips, 1987; Feuerstein et al., 1987; Revenson and Felton, 1989). It should be noted that in this explanation a kinesiological mechanism (muscle weakness) mediates between psychological processes (anxiety, coping, and avoidance) and symptoms in OA (pain and disability). The findings of Lunghi et al. (1978), described above, give some support to this explanation.

\section{DISCUSSION}

\section{Summary of Findings and Explanatory Mechanisms}

This review of behavioral mechanisms of pain and disability in OA can be summarized as follows:

Articular Level. Radiological evidence of OA has a distinct relationship to pain and disability, at least in population-based studies. This applies 
to all joints which have been studied, except the spine. However, the relationship is far from perfect. The weakness of this relationship seems to be explained by a discrepancy between the nociceptive processes and the articular changes detected by means of radiological assessment. Cartilage degeneration is (indirectly) assessed by means of a radiograph, but it does not directly contribute to nociception. Only degeneration of subchondral bone is both visible on a radiograph and also a contribution to nociception.

Kinesiological Level. (a) Muscle weakness is clearly associated with pain and disability in OA. A biomechanical model seems to offer the best explanation for this finding. According to this model muscle weakness leads to unstable joints; stress on unstable joints results in pain and disability. Avoidance of pain-related activities enhances muscle weakness, which starts a vicious circle of avoidance, muscle weakness, pain, and disability. (b) An association between restricted range of joint motion and disability has been demonstrated. Both capsular and muscular mechanisms may explain the restricted range of joint motion and disability in $\mathrm{OA}$.

Psychological Level. (a) Anxiety, coping style, and possibly depression are associated with pain and (observed) disability in OA. Both cognitive and behavioral mechanisms have been proposed to account for these associations. According to the cognitive mechanisms, emotions and symptoms are integrated into a cognitive schema. As a result, emotional experiences activate the experience of symptoms, and vice versa. This mechanism fails to explain the negative association between coping style and (observed) disability in OA. A behavioral mechanism is better suited to explain the latter association. Accordingly, an emotional or catastrophizing reaction to pain is hypothesized to strengthen the patient's tendency to avoid pain-related activities; such avoidance enhances muscle weakness, which leads to unstable joints, pain, and disability. (b) It has been found that a decrease in life events is followed by an increase in OA symptoms. A lack of distraction might explain this association: In the absence of distracting events patients probably focus on their symptoms which enhances their suffering.

\section{Critical Remarks and Suggestions for Further Research}

A number of critical remarks apply to the research reviewed above. These remarks concern both methodological and conceptual issues.

(i) The studies of the kinesiological effects on pain and disability have failed to control for the level of articular degeneration (radiological OA). Consequently, it is not clear to what extent kinesiological mechanisms separately contribute to pain and disability. Theoretically it is possible that 
kinesiological degeneration (reduced muscle strength, etc.) is perfectly correlated with articular degeneration. Although clinical experience suggests that this is not true, controlled evidence to refute this hypothesis is not available.

This criticism does not apply to the studies on psychological determinants of pain and disability. All studies of psychological determinants, except one (Lunghi et al, 1978), controlled for the level of articular degeneration. Psychological processes are associated with pain and disability in OA, irrespective of the level of articular degeneration. However, the studies on psychological determinants did not control for the kinesiological determinants. It is consequently not clear to what extent psychological and kinesiological determinants of pain and disability operate independently of one another.

To determine the distinct contribution of articular, kinesiological, and psychological processes to pain and disability in OA, these processes should be assessed altogether in one study. It is suggested that such a study be done in the future.

(ii) The review of the studies of articular determinants of pain indicated significant associations in population-based studies, but such associations were largely absent in studies of patients. It was argued that in studies of patients the range of pain complaints is probably limited, which makes it difficult to demonstrate significant associations. The studies on kinesiological and psychological determinants almost all have used patients as subjects. Although significant associations have been found in these studies, it is quite possible that the strength of these associations has been underestimated because of a limited range of pain complaints. It is suggested that in future research steps are taken to ensure inclusion of subjects with a wide range of pain and disability.

Many studies in this review failed to document the level and the range of pain, disability, and other important variables. This information is of vital importance for the interpretation of results. It is therefore suggested that future reports clearly document the distribution of important variables such as pain.

(iii) The review indicated deficiencies concerning the measurement of pain and psychological variables. In several studies pain has been assessed in an unstandardized way. If such a study shows a lack of significant findings, this may be due to the inadequate assessment of pain. Therefore, it is advisable to use properly standardized tests. In order to allow a straightforward interpretation of findings, the test's reliability, validity, and sensitivity to differences in pain should have been demonstrated.

In certain tests assessing psychological status, somatic disease manifestations and psychological status are confused. This applies in particular 
to tests assessing depression. Items reflecting somatic disease should not be present in tests of psychological status. Alternatively, these items should be removed from the test (Pincus et al., 1982; Peck et al., 1989).

Guccione et al. (1990) have demonstrated that the criteria used to define the presence of OA (e.g., using grade 2 versus 3 as the cut off score) affect the strength of the association of radiological OA and disability. It was shown that the strength of the association depends on which aspect of disability is being measured (e.g., stair climbing, walking a mile, or global disability). These authors concluded that inclusion of minor radiological signs and a global assessment of disability may cause nonsignificant or weak correlations. Future studies should use specific definitions of $O A$ and report the impact of specific severity levels of disease on particular functional disabilities.

(iv) The majority of the studies in this review have used cross-sectional designs. With a cross-sectional design it is not possible to interpret the data in terms of cause and effect. Experimental or prospective designs are required in order to allow conclusions on causal determinants of pain and disability in OA. This applies in particular to kinesiological and psychological determinants. It is certainly not self-evident that kinesiological and psychological processes are causal determinants of pain and disability in $\mathrm{OA}$. Research using an experimental or prospective design is urgently needed in these areas.

(v) Finally, many studies in this review lack a sound conceptual framework. Theoretical accounts of the association among determinants, pain, and disability are frequently absent. Authors report on the association of certain determinants, pain, and disability, but they fail to provide an adequate explanation of the observed associations. In this review, articular, kinesiological, and psychological mechanisms which explain the observed associations have been discussed. It is hoped that this will contribute to an improvement of the conceptual framework of future research.

Two important and promising areas of research seem to emerge form this review. First, processes at the kinesiological level seem to be important determinants of pain and disability in OA. Reductions of muscle strength and of joint motion have been studied, but more elaborate and betterdesigned research on these mechanisms is appropriate. In addition, research on other kinesiological mechanisms is indicated. This applies, for example, to muscle spasm as a determinant of pain, which has not yet been studied in OA. Second, psychological processes possibly affect symptoms in OA by way of kinesiological processes. For example, the effect of catastrophizing on pain and disability might be mediated by way of avoidance of pain-related activities and the ensuing muscle weakness. But direct evidence in support of this mechanism is not available. Elucidation of these 
mediating mechanisms will contribute to the understanding of psychological effects on pain and disability in OA.

\section{REFERENCES}

Acheson, R. M., and Ginsburg, G. N. (1973). New Haven survey of joint diseases. XVI. Impairment, disability and arthritis. Br. J. Prev. Soc. Med. 27: 168-176.

Acheson, R. M., Chan, Y. K., and Clemett, A. R. (1970). New Haven survey of joint diseases. XII. Distribution and symptoms of osteoarthrosis in the hands with reference to handedness. Ann. Rheum. Dis. 29: 275-286.

Badley, E. M., Wagstaff, S., and Wood, Ph. H. N. (1984). Measures of functional ability (disability) in arthritis in relation to impairment of range of joint movement. Ann. Rheum. Dis. 43: 563-569.

Baron, M., Dutil, E., Berkson, L., Lander, Ph., and Becker, R. (1987). Handfunction in the elderly: Relation to osteoarthritis. Arth. Rheum. 14: 815-819.

Barksy, A. J., and Klerman, G. L. (1983). Overview: Hypochondriasis, bodily complaints and somatic styles. Am. J. Psychiat. 140: 273-283.

Beals, C., Lampman, R. M., Banwell, B. F., Braunstein, E. M., Albers, J. W., and Castor, C. W. (1985). Measurement of exercise tolerance in patients with rheumatoid arthritis or osteoarthritis. J. Rheumatol. 12: 458-461.

Brandt, K .D. (1985a). Degenerative joint disease and other primary diseases in cartilage. In Kelley, W. N., Harris, E. D., Rudy, S., and Sledge, C. B. (eds.), Textbook of Rheumaiology, 2nd ed., Saunders, Philadelphia, pp. 1417-1431.

Brandt, K. D. (1985b). Osteoarthritis: Clinical patterns and pathology. In Kelley, W. N., Harris, E. D., Ruddy, S., and Sledge, C. B. (eds.), Textbook of Rheumatology, 2nd ed., Saunders, Philadelphia, pp. 1432-1447.

Cobb, S., Merchant, W. R., and Rubin, T. (1957). The relationship of symptoms to osteoarthritis. J. Chron. Dis. 5: 197-204.

Davis, M. A. (1981). Sex differences in reporting osteoarthritic symptoms: A sociomedical approach. J. Health Soc. Behav. 22: 298-310.

Doice, J. J., and Raczynski, J. M. (1985). Neuromuscular activity and electromyography in painful backs: Psychological and biomechanical models in assessment and treatment. Psychol. Bull. 97: 502-520.

Ekdahl, C., Andersson, S., and Svensson, B. (1989). Muscle function of the lower extremities in rheumatoid arthritis and osteoarthritis. J. Clin. Epidemiol. 42: 947-954.

Felson, D. T., Maimark, A., Anderson, J., Kazis, L., Castelli, W., and Meenan, R. F. (1987). The prevalence of knee osteoarthritis in the elderly. Arth. Rheum. 30: 914-918.

Feuerstein, M., Papciak, and A. S., and Hoon, P. E. (1987). Behavioral mechanisms of chronic low back pain. Clin Psychol. Rev. 7: 243-273.

Flor, H., and Turk, D. C. (1984). Etiological theories and treatments for chronic back pain. I. Somatic models and interventions. Pain 19: 105-121.

Forman, M. D., Malamet, R., and Kaplan, D. (1983). A survey of osteoarthritis of the knee in the elderly. J. Rheumatol. 10: 282-287.

Gresham, G. E., and Rathey, U. K. (1975). Osteoarthritis in knees of aged persons: relationship between roentgenographic and clinical manifestations, JAMA 233: 168-170.

Guccione, A. A., Felson, D. T., and Anderson, J. J. (1990). Defining arthritis and measuring functional status in elders: Methodological issues in the study of disease and physical disability. Am. J. Public Health 80: 945-949.

Keefe, F. J., and Gill, K. M. (1986). Behavioral concepts in the analysis of chronic pain syndromes. J. Consult. Clin. Psychol. 54: 776-783.

Keefe, F. J., Caldwell, D. S., Queen, K. T., Gil, K. M., Martinez, S., Crisson, J. E. Ogden, W., and Nunley, J. (1987a). Pain coping strategies in osteoarthritis patients. J. Consult. Clin. Psychol. 55: 208-212. 
Keefe, F. J., Caldwell, D. S., Queen, K., Gil, K. M., Martinez, S., Crisson, J. E., Ogden, W., and Nunley, J. (1987b). Osteoarthritic knee pain: A behavioral analysis. Pain 28: 309-321.

Kellgren, J. H. (1983). Pain in osteoarthritis. J. Rheum. (Suppl. 9) 10: 108-109.

Kellgren, J. H., and Lawrence, J. S. (1952). Rheumatism in miners. Part II: X-ray study. Br. J. Industr. Med. 9: 197-207.

Kellgren, J. H., and Lawrence, J. S. (1957). Radiological assessment of osteoarthrosis. Ann. Rheum. Dis. 16: 494-502.

Kellgren, J. H., and Lawrence, J. S. (1963). Atlas of Standard Radiographs, prepared by Department of Rheumatology and Medical Illustrations, University of Manchester, Blackwell, Oxford.

Kirwan, J. R., and Silman, A. J. (1987). Epidemiological, sociological and environmental aspects of rheumatoid arthritis and osteoarthritis. Baillieres Clin. Rheumatol. 1: 467-489.

Lankhorst, G. J., Van de Stadt, R. J., Van der Korst, J. K., Hinlopen-Bonrath, E., Griffioen, F. M. M., and De Boer, W. (1982). Relationship of isometric knee extension torque and functional variables in osteoarthritis of the knee. Scand. J. Rehab. Med. 14: 7-20.

Lankhorst, G. J., Van de Stadt, R. J., and Van der Korst, J. K. (1985). The relationships of functional capacity, pain and isometric and isokinetic torque in osteoarthritis of the knee. Scand. J. Rehab. Med. 17: 167-172.

Lawrence, J. S., Bremmer, J. M., and Bier, F. (1966). Osteoarthritis: Prevalence in the population and relationship between symptoms and x-ray changes. Ann. Rheum. Dis. 25: $1-24$.

Lichtenberg, P. A., and Skehan, M. W., and Swensen, C. H. (1984). The role of personality, recent life stress and arthritic severity in predicting pain. J. Psychosom. Res. 28: 231-236.

Lorig, K. Konkol, L., and Gonzalez, V. (1987). Arthritis patient education: A review of the literature. Pat. Educ. Couns. 10: 207-252.

Lunghi, M. E., Miller, P. McC, and McQuillan, W. M. (1978). Psychological factors in osteoarthritis of the hip. J. Psychosom. Res. 22: 57-63.

Massardo, L., Watt, I., Cushnaghan, J., and Dieppe, P. (1989). Osteoarthritis of the knee joint: An eight year prospective study. Ann. Rheum. Dis. 48: 893-897.

McCaul, K. D., and Malott, J. M. (1984). Distraction and coping with pain. Psychol. Bull. 95: 516-533.

Miller, R., Kettelkamp, D., Laubenthal, K. N., Karagiorgos, A., and Smidt, G. L. (1973). Quantitative correlations in degenerative arthritis of the knee. J. Bone Joint Surg. 55: 956-962.

Minor, M. A., Hewett, J. E., Webel, R. R., Dreisinger, T. E., and Kay, D. R. (1988). Exercise tolerance and disease related measures in patients with rheumatoid arthritis and osteoarthritis. J. Rheumatol. 15: 905-911.

Moskowitz, R. W. (1985). Clinical and laboratory findings in osteoarthritis. In McCarthy, P. J. (ed.), Arthritis and Allied Conditions, 10th ed., Lea and Febiger, Philadelphia, pp. 1408-1431.

Nordesjö, L. O., Nordgren, B., Wigren, A., and Kolstad, K. (1983). Isometric strength and endurance in patients with severe rheumatoid arthritis or osteoarthritis in the knee joints. Scand. J. Rheumatol. 12: 152-156,

Patrick, M., Aldridge, S., Hamilton, E., Manhire, A., and Doherty, M. (1989). A controlled study of hand function in nodal and erosive osteoarthritis. Ann. Rheum. Dis. 48: 978-982.

Peck, J. R., Smith, T. W., Ward, J. R., and Milano, R. (1989). Disability and depression in rheumatoid arthritis. Arth. Rheum. 32: 1100-1106.

Pennebaker, J. W. (1982). The Psychology of Physical Symptoms, Springer, New York.

Philips, H. C. (1987). Avoidance behavior and its role in sustaining chronic pain. Behav. Res. Ther. 25: 273-279.

Pincus, T., Callahan, L. F., Bradley, L. A., Vaughn, W. K., and Wolfe, F. (1986). Elevated MMPI scores for hypochondriasis, depression and hysteria in patients with rheumatoid arthritis reflect disease rather than psychological status. Anth. Rheum. 29: 1456-1466.

Pincus, T., Mitchell, J. M., and Burkhauser, R. V. (1989). Substantial work disability and earnings losses in individuals less than age 65 with osteoarthritis: Comparisons with rheumatoid arthritis. J. Clin. Epidemiol. 42: 449-457. 
Revenson, T. A., and Felton, B. J. (1989). Disability and coping as predictors of psychological adjustment to rheumatoid arthritis. J. Consult. Clin. Psychol. 57: 344-348.

Steinbrocker, O., Traegger, C., and Batterman, R. (1948). Therapeutic criteria in rheumatoid arthritis. JAMA 140: 659-662.

Stokes, M., and Young. A. (1984). The contribution of reflect inhibition to arthrogenous muscle weakness. Clin. Sci. 67: 7-14.

Summers, M. N., Haley, W. E., Reveille, J. D., and Alarcon, G. S. (1988). Radiographic assessment and psychologic variables as predictors of pain and functional impairment in osteoarthritis of the knee or hip. Arth. Rheum. 31: 204-209.

Threlkeld, A. J., and Currier, D. P. (1988). Osteoarthritis: Effects on synovial joint tissues. Phys. Ther. 68: 364-370.

Turk, D. C., and Flor, H. (1984). Etiological theories and treatments for chronic back pain. II. Psychological models and interventions. Pain 19: 209-233.

Watson, D., and Pennebaker, J. W. (1989). Health complaints, stress and distress: Exploring the central role of negative affectivity. Psychol. Rev. 96: 234-254.

Yelin, E., Lubeck, D., Holman, H., and Epstein, W. (1987). The impact of rheumatoid arthritis and osteoarthritis: The activities of patients with rheumatoid arthritis compared to controls. J. Rheumatol. 14: 710-717. 